\title{
Rapid Signalling Mechanisms in the Cardiovasculo-Protective Effects of Estrogen
}

\author{
Richard Heads ${ }^{1}$, Ana-Roberta Niță², Greg A Knock ${ }^{2,3}$, and Richard J Heads ${ }^{2,4}$ \\ ${ }^{1}$ Affiliation not available \\ ${ }^{2}$ School of Bioscience Education, Faculty of Life Sciences and Medicine, King's College \\ London \\ ${ }^{3}$ School of Immunology and Microbial Sciences, Faculty of Life Sciences and Medicine, \\ King's College London \\ ${ }^{4}$ Cardiovascular Research Section, King's BHF Centre of Research Excellence, School of \\ Cardiovascular Medicine and Sciences, Faculty of Life Sciences and Medicine, BHF Centre \\ of Research Excellence, School of Cardiovascular Medicine and Sciences, Faculty of Life \\ Sciences and Medicine, King's College London
}

October 12, 2020

\begin{abstract}
In modern society, cardiovascular disease remains the biggest single threat to life, being responsible for approximately $1 / 3$ of worldwide deaths. Male prevalence is significantly higher than that of women until after menopause, when the prevalence of CVD increases in females until, over the age of 80 , it eventually exceeds that of men. Because of the coincidence of CVD prevalence increasing after menopause, the role of estrogen in the cardiovascular system has been intensively researched during the past 2 decades in vitro, in vivo and inobservational studies. Most of these studies suggested that endogenous estrogen confers cardiovascular protective and anti-inflammatory effects. However, clinical studies of the cardioprotective effects of hormone replacement therapies (HRT) not only failed to produce proof of protective effects, but also revealed the potential harm estrogen could cause. The "critical window of hormone therapy" hypothesis affirms that the moment of its administration is essential for positive treatment outcomes, pre-menopause (3-5 years before menopause) and immediate post menopause being thought to be the most appropriate time for intervention. Since many of the cardioprotective effects of estrogen signaling are mediated by effects on the vasculature, this review aims to discuss the effects of estrogen on vascular smooth muscle cells (VSMCs) and endothelial cells (ECs) with a focus on the role of estrogen receptors (ER $\alpha$, ER $\beta$ and GPER) in triggering the more recently discovered rapid or membrane delimited (non-genomic) signaling cascades that are vital for regulating the vascular tone, preventing hypertension and other cardiovascular diseases.
\end{abstract}

\section{Hosted file}

ER Review_Nita_Knock_HEADS.pdf available at https://authorea.com/users/357590/articles/ 486345-rapid-signalling-mechanisms-in-the-cardiovasculo-protective-effects-of-estrogen 